\title{
Markers of MEK inhibitor resistance in low-grade serous ovarian cancer: EGFR is a potential therapeutic target
}

\author{
Marta Llaurado Fernandez ', Amy Dawson', Joshua Hoenisch', Hannah Kim', Sylvia Bamford', \\ Clara Salamanca2 , Gabriel DiMattia 3,4, Trevor Shepherd ${ }^{3,4}$, Mattia Cremona ${ }^{5}$, Bryan Hennessy ${ }^{5}$, \\ Shawn Anderson ${ }^{6}$, Stanislav Volik ${ }^{6}$, Colin C. Collins ${ }^{6}$, David G. Huntsman ${ }^{2,7}$ and Mark S. Carey ${ }^{1,8^{*}}$
}

\begin{abstract}
Background: Although low-grade serous ovarian cancer (LGSC) is rare, case-fatality rates are high as most patients present with advanced disease and current cytotoxic therapies are not overly effective. Recognizing that these cancers may be driven by MAPK pathway activation, MEK inhibitors (MEKi) are being tested in clinical trials. LGSC respond to MEKi only in a subgroup of patients, so predictive biomarkers and better therapies will be needed.

Methods: We evaluated a number of patient-derived LGSC cell lines, previously classified according to their MEKi sensitivity. Two cell lines were genomically compared against their matching tumors samples. MEKi-sensitive and MEKi-resistant lines were compared using whole exome sequencing and reverse phase protein array. Two treatment combinations targeting MEKi resistance markers were also evaluated using cell proliferation, cell viability, cell signaling, and drug synergism assays.

Results: Low-grade serous ovarian cancer cell lines recapitulated the genomic aberrations from their matching tumor samples. We identified three potential predictive biomarkers that distinguish MEKi sensitive and resistant lines: KRAS mutation status, and EGFR and PKC-alpha protein expression. The biomarkers were validated in three newly developed LGSC cell lines. Sub-lethal combination of MEK and EGFR inhibition showed drug synergy and caused complete cell death in two of four MEKi-resistant cell lines tested.
\end{abstract}

Conclusions: KRAS mutations and the protein expression of EGFR and PKC-alpha should be evaluated as predictive biomarkers in patients with LGSC treated with MEKi. Combination therapy using a MEKi with EGFR inhibition may represent a promising new therapy for patients with MEKi-resistant LGSC.

Keywords: Ovarian cancer, MEK inhibitor, EGFR inhibitor, PKC-alpha, Predictive biomarkers

\section{Background}

Each year in Canada and the United States, over 25,000 women are diagnosed with ovarian cancer [1, 2]. Lowgrade serous ovarian cancer (LGSC) accounts for 5-10\% of these cancers [3, 4], affecting approximately 2000 women per year. This rare form of ovarian cancer is often diagnosed in pre-menopausal women and frequently

\footnotetext{
${ }^{*}$ Correspondence: mark.carey@ubc.ca

${ }^{8}$ Division of Gynecologic Oncology, Diamond Health Centre, 2775 Laurel

St., 6th Floor, Vancouver, BC V5Z 1M9, Canada

Full list of author information is available at the end of the article
}

found in advanced stages. Although LGSC is considered to be a less aggressive subtype than other ovarian cancers, response rates to chemotherapy are low, ranging from 4 to $25 \%$ [5]. Consequently, long-term fatality rates are high with only $10-20 \%$ of women surviving 10 years after diagnosis $[5,6]$.

It is now recognized that LGSC has unique clinical, pathological, and molecular characteristics compared to other types of ovarian cancers, such as the high-grade serous ovarian carcinoma (HGSC) [7, 8]. Molecular studies performed on LGSC tumors revealed that mutations in the TP53 gene are rare $(8 \%$ in LGSC versus $96 \%$ in 
HGSC) $[9,10]$, and that expression of estrogen (ER) and progesterone (PR) receptors is frequently observed [11, 12]. LGSC is also characterized by activation of the mitogen-activated protein kinase (MAPK) pathway. Mutations affecting this pathway are seen in KRAS (20-40\%), NRAS (7-26\%) and BRAF (5-33\%) genes [13-20]. Evidence of MAPK pathway activation in LGSC [21] led to a key clinical trial evaluating the efficacy of the MEK inhibitor (MEKi) selumetinib for the treatment of patients with advanced and/or recurrent LGSC (GOG-0239). The results from this trial, published in 2013, shown a $15 \%$ response rate and $65 \%$ disease stabilization [22]. A second clinical trial of the MEKi binimetinib (MILO trial, NCT01849874) was closed at the interim analysis in 2016, because it did not show the anticipated predefined benefits on progression-free survival (PFS). Despite these unexpected results, durable responses to binimetinib were observed in LGSC with MAPK pathway alterations [23]. Currently, an international randomized phase II/III clinical trial using the MEKi trametinib is ongoing (NCT02101788) and a translational research component to better understand the molecular mechanisms of MEKi efficacy is included.

To date, preclinical laboratory research in LGSC has been limited to tumor tissues. The low frequency and slow growth rate of these tumors have challenged the development of cell lines and animal xenograft models. In the past 5 years, our laboratory has successfully established a collection of patient-derived LGSC cell lines that are now available for pre-clinical drug testing. Previously, we evaluated the effects of four different MEKi (selumetinib, trametinib, binimetinib, refametinib) in eight advanced/recurrent LGSC cell lines. Our results indicated that there were substantial differences in cellular response and on-target drug efficacy between cell lines and drugs [24]. Encouraged by promising results from MEKi clinical trials in a subset of LGSC patients, we sought to identify biomarkers that could predict response to treatment using LGSC cell lines, by comparing the proteogenomic profiles of MEKi-sensitive (MEKi-Se) and MEKi-resistant (MEKi-Re) LGSC cell lines, and subsequently evaluating the potential therapeutic value of two proteins (EGFR and PKC-alpha) associated with MEKi resistance.

\section{Materials and methods}

\section{Tumor samples and clinical information}

Advanced or recurrent LGSC samples (tumor and ascites) were obtained from the OvCaRe gynecologic tumor bank (Vancouver General Hospital/British Columbia Cancer Agency (BCCA), and the John and Mary Knight Translational Ovarian Cancer Research Unit (London Regional Cancer Program, London, Ontario, Canada). Tumor bank protocols, cell line derivation, and all research relating to this study was approved by institutional human ethics review boards at BCCA (H14-02859), the University of British Columbia (UBC; R05-0119), and the University of Western Ontario (HSREB 12668E). Clinical information was extracted retrospectively from patient records. Tumor histology was confirmed by a gynecological pathologist.

\section{Patient-derived LGSC cell lines}

Low-grade serous ovarian cancer patient-derived cell lines were established through continuous in vitro culture of patient material obtained through OvCaRe or the John and Mary Knight Translational Ovarian Cancer Research Unit (cell line iOvCa241) tumor banks. Cultures were established and maintained in M199:MCDB105 (1:1) media (Cat. No. M5017 and M6395, Sigma-Aldrich, Oakville, Ontario, Canada) supplemented with 10\% defined fetal bovine serum (dFBS; Cat. No. SH30070.03, Hyclone, GE Life Sciences, Logan, UT, USA) maintained at $37{ }^{\circ} \mathrm{C}$ and $5 \% \mathrm{CO}_{2}$. No immortalization methods were used. Doubling time of these cells ranged from 30 to $80 \mathrm{~h}$, with an average of $47 \mathrm{~h}$, reflecting the clinical slow growth rate of LGSC.

\section{Sample authentication (cell line, tumor, buffy coat)}

Microsatellite analysis of short tandem repeats (STRs) was performed on LGSC cell lines and corresponding tumor and buffy coat samples for cell line authentication. STR analyses of 10 loci were performed by Genewiz Inc. (South Plainfield, NJ) (Data available upon request). STR results confirm that all LGSC cell lines and buffy coat samples match to corresponding tumor samples.

\section{Genome sequencing}

Whole exome sequencing (WES): Agilent SureSelect RNA Library All Exons v6 protocol was performed by Beijing Genome Institute, per manufacturer's guidelines. Quality and quantity of post-capture libraries were assessed using an Agilent 2100 Bioanalyzer. Libraries were sequenced on an Illumina Hiseq 4000 (PE 100). Copy number variation (CNV) analysis: Data analysis was performed using Nexus Copy Number Discovery Edition Version 9.0 (BioDiscovery, Inc., El Segundo, CA). Samples were processed using the Nexus NGS functionality (BAM ngCGH) with the FASST2 segmentation. The log ratio thresholds for single copy gain and single copy loss were set at +0.18 and -0.18 , respectively. The log ratio thresholds for gain of 2 or more copies and for a homozygous loss were set at +0.6 and -1.0 , respectively. Tumor sample BAM files were processed with corresponding normal tissue BAM files. Probes were normalized to median. Mutation analysis: Sequence alignment and 
mutation calling were performed in Partek Flow environment (๔ 2017 Partek Inc). Sequence reads were aligned to GRCh38/hg38 human genome build using bwa 0.7.2. Variants were called using Strelka 1.0.15 for all cell lines except for VOA-1312 (lacking buffy coat sample). VOA1312 variant calling was performed using LoFreq 2.1.3.a. The called variants were annotated using the wAnnovar software (reference obtained from: http://jmg.bmj.com/ content/49/7/433.citation-tools). Annotated calls were then filtered to show only protein-changing SNVs that were present in cell line DNA at allele frequencies (AF) greater than 0.1 and coverage higher than $16 \times$. For VOA1312, all calls not present in dbSNP (version 138) were retained, while of the calls that were present in dbSNP, only calls with (average heterozygocity + aveHet standard error) $<0.1$ were retained. These were additionally filtered using the same criteria as for the Strelka calls.

Whole genome sequencing (WGS): Genomic data from LGSC tumors T7 and T11 were obtained from the personalized oncogenomics (POG) program at the BCCA. Methodology has been previously described in detail [25]. To summarize, genome and transcriptome libraries were sequenced on HiSeq instruments (Illumina, San Diego, California) using V3 or V4 chemistry and pairedend 150 or 125 base reads, respectively. Target depth was $80 \times$ coverage for the tumor genome and $40 \times$ for the normal genome.

\section{Cell proliferation assays}

Assessment of MEKi sensitivity using trametinib (GSK1120212; Sellekchem, Cat. No. S2673) and selumetinib (AZD6244; Cat. No. S1008), were performed as previously described [24]. Cell proliferation was monitored using IncuCyte ${ }^{\mathrm{TM}}$ real-time imaging technology using a non-labeled monolayer confluence approach (Essen Biosciences, Ann Arbor, MI, USA). LGSC cell lines were plated at $15-20 \%$ confluence in 96 -well plates. After $24 \mathrm{~h}$, cells were treated once with DMSO (control) or differing drug concentrations [erlotinib alone $(10 \mu \mathrm{M}$ and $2.5 \mu \mathrm{M})$, in combination $(10,5,2.5,1.25$, and $0.63 \mu \mathrm{M})$, high and low doses of MEKi treatment $(1 \mu \mathrm{M}$ and $0.5 \mu \mathrm{M}$ selumetinib; $0.1 \mu \mathrm{M}$ and $0.05 \mu \mathrm{M}$ trametinib; doses for preclinical MEKi assays as previously published)] [24]. Trametinib and selumetinib were selected as the MEKi for combination treatments. These two drugs are most commonly used clinically for treating LGSC, and binimetinib may lack efficacy based on results from the MILO clinical trial (NCT01849874). Drug doses of selumetinib and trametinib were chosen based on IC50 results from our previous experiments [24]. Selected concentrations for these experiments are in keeping with steady state serum levels (selumetinib $2 \mu \mathrm{M}$ and trametinib $30 \mathrm{nM}$ ) reported for these drugs in humans $[26,27]$. Phase contrast images of cells were taken every 6-h for 4-5 days. Each condition was evaluated using four technical replicates and experiments were repeated for verification. Data analysis was performed using IncuCyte ${ }^{\mathrm{TM}}$ software. Statistical analyses using the $t$-test on the final time point values of each assay were performed to compare treatment conditions. Differences were considered significant at a p-value $<0.05$.

\section{Cell viability assay}

Cell viability was measured using MTS-Cell Titer 96R Aqueous Non-Radioactive Cell Proliferation Assay, following manufacturer recommendations (Cat. No. G5430, Promega, Madison, WI, USA) at endpoint of Incucyte ${ }^{\mathrm{TM}}$ proliferation assays. Treatment media was replaced with $100 \mu \mathrm{L}$ of fresh media and $20 \mu \mathrm{L}$ of MTS. Plates were incubated for $3.5 \mathrm{~h}$ at $37{ }^{\circ} \mathrm{C}$ in humidified $5 \% \mathrm{CO}_{2}$. Absorbance at $490 \mathrm{~nm}$ was measured using a microplate reader (BioTek Epoch SN257811). Viability for each treatment was compared to DMSO treated cells. Wells were subsequently stained with crystal violet $(\mathrm{CV})$ to determine residual cells after treatment. Statistical analysis using t-test were used to compare treatment conditions and differences were considered significant at a p-value $<0.05$.

\section{IC50 determination}

Erlotinib (Cat. No. S7786) were purchased from Selleck Chemicals (Houston, TX, USA). Dimethylsulfoxide (DMSO; Sigma, Cat. No. D2650) was purchased from Sigma-Aldrich (Oakville, Ontario, Canada). Cells were seeded in 96 -well plates at $40-50 \%$ confluence and treated after $24 \mathrm{~h}$ with DMSO or a range of drug concentrations. The inhibitory concentration (IC50, representing $50 \%$ of total cell viability) was determined using crystal violet staining after $72 \mathrm{~h}$ drug treatment.

\section{Western blot analysis}

Cell lysates were prepared according to previously published protocols [24], then $20 \mu \mathrm{g}$ samples were separated on an $8 \%$ SDS-PAGE gel, transferred to nitrocellulose membranes and probed with primary antibodies including ERK1/2 (Millipore, Cat. No. 06-182), p-MAPK (p-ERK1/2, Cell Signaling, Cat. No. 4376S), MEK1/2 (Cell Signaling, Cat. No. 9122), p-MEK1/2 (Cell Signaling, Cat. No. 9154), PKC-alpha (Cell Signaling, Cat. No. 2056), EGFR (Santa Cruz, Cat. No. 71032), p-EGFR (Cell Signaling, Cat. No. 2234), PARP (Cell Signaling, Cat. No. 9542), and c-PARP (Cell Signaling, Cat. No. 9541S). Vinculin (V9131, Sigma) was used as a protein loading control. Horseradish peroxidase (HRP)-conjugated secondary antibodies (goat-anti-mouse or goat-anti-rabbit, Sigma Cat. No. A9917 and A0545) were used accordingly. 
Western blots were imaged using Immobilon HRP reagent (Cat. No. WBKLS0500, Millipore, Etobicoke, ON, Canada) and developed by autoradiograph.

\section{Reverse-phase protein array (RPPA) analysis}

Reverse-phase protein array on whole tumor and cell line lysates was performed as previously described [28, 29]. Proteomic profiles of 8 LGSC cell lines, 2 MEKi-sensitive (VOA-1312, iOvCa241) and 6 MEKi-resistant (VOA1056, VOA-3993; VOA-3448, VOA-3723; VOA-4627, VOA-4698), were analyzed. LGSC cells were treated for $24 \mathrm{~h}$ with $1 \mu \mathrm{L} / \mathrm{mL}$ DMSO or MEKi (trametinib $0.1 \mu \mathrm{M}$, selumetinib $1.0 \mu \mathrm{M})$ in biological triplicate as previously described $[24,30]$. Antibodies $(n=91)$ against cell surface growth factor receptors, common signaling pathway proteins, steroid hormone receptors, and other proteins involved in proliferation and apoptosis were used (Additional file 1: Table S1). Data was analyzed using SPSS software (Version 20, Chicago, Illinois). Differentially expressed proteins between cell lines and treatment conditions were determined using the t-test [31]. The MannWhitney $U$ test was used for proteins with non-normally distributed expression levels. False discovery rates were not calculated as putative markers were validated by western blot.

\section{shRNA-mediated knockdown of PKC-alpha expression (PRKCA gene)}

shERWOOD-UltramiR shRNA lentiviral target gene set containing three PRKCA shRNA sequences and one non-target shRNA (Cat. No. TLHVU1401-5578) was purchased from transOMIC Technologies (Huntsville, AL). VOA-3723 and VOA-6406 were plated at 50\% confluence in 6-well tissue culture dishes $24 \mathrm{~h}$ prior to lentiviral transduction. 199:105 media supplemented with $1 \%$ Hyclone dFBS and polybrene $(2 \mu \mathrm{g} / \mathrm{mL}$ for VOA3723, $0.5 \mu \mathrm{g} / \mathrm{mL}$ for VOA-6406) and lentivirus expressing non-targeting shRNA or PRKCA shRNA (multiplicity of infection $[\mathrm{MOI}]=26$ for VOA-3723, MOI $=1.5$ for VOA-6406) in a total volume of $1.5 \mathrm{~mL}$ was added. After $24 \mathrm{~h}$, cells were washed with PBS and complete media was added. Successful transduction was confirmed using confocal microscopy. After an additional 24-h recovery, transduced LGSC cells were selected and maintained using puromycin $(1.0 \mu \mathrm{g} / \mathrm{mL}$ for VOA3723, $0.5 \mu \mathrm{g} / \mathrm{mL}$ for VOA6406).

\section{Drug synergy analysis}

Cell proliferation, viability and crystal violet results from in vitro drug testing (single drug and drug combinations) were used to assess drug synergism using CompuSyn software (http://www.combosyn.com). This software is based on the median-effect principle and the combination index-isobologram theorem (Chou-Talalay) [32]. Drug doses (D) and effects (fa) were entered (nonconstant ratios) for single drug doses and combinations, and combination indices (CI) were generated. The CI values quantitatively defined synergism $(\mathrm{CI}<1)$, additive effect $(C I=1)$, and antagonism $(C I>1)$.

\section{Results \\ Development and MEKi treatment evaluation of LGSC cell lines}

Our laboratory previously established a collection of LGSC cell lines derived from patients with advanced/ recurrent disease. Preclinical evaluation of four MEKi in eight different LGSC cell lines resulted in the identification of two distinct phenotypes: MEKi-sensitive (MEKiSe) cell lines $(n=2)$, and MEKi-resistant (MEKi-Re) cell lines $(n=6)$. In this first study, MEKi drug concentrations and IC50 values were reported [24]. Recognizing the challenges using IC-50 values to assess drug efficacy invitro, we established a stringent definition of MEKi sensitivity/resistance recognizing that only $15 \%$ of patients with advanced/recurrent LGSC will show tumor experience regression when treated with a MEKi. Thus, we classified cell lines as MEKi-Se if a single dose of MEKi resulted in complete cell death over a period of 5 days. Alternatively, cell lines were considered MEKi-Re if they continued to proliferate (even despite some degree of inhibition) under the same treatment conditions. Continuing our previous work, we established three new LGSC cell lines from three independent patients (VOA-6406, VOA-8862, VOA-9164). Tumor cells from three other LGSC patients were also grown temporarily as primary cultures (VOA-6800, VOA-6857, VOA-7604). Using our previous classification criteria, two of these new lines were classified as MEKi-Se (VOA-9164 and VOA-8862), and one as MEKi-Re (VOA-6406-see Additional file 2: Figure S1). STR analysis confirmed unique microsatellite profiles for each of these lines, matching the profiles of the original tumor tissues from which they were derived (data available upon request).

\section{Genomic characterization of MEKi-Se and MEKi-Re LGSC cells}

WES was performed to characterize the genomic profiles of our LGSC cell lines and primary cultures. First, we compared the copy number profiles of two of our cell cultures (VOA-4627, VOA-6857) with those of their associated tumor samples (from WGS data). As shown in Fig. 1, the copy number variation profiles of the paired samples showed a very high degree of correlation. Of note, VOA-4627 line was derived from an ascites sample taken 2 years after the tumor sample collected previously at cytoreductive surgery. 
(See figure on next page.)

Fig. 1 Comparison of genomic profiles between two LGSC cell cultures and their associated LGSC tumor samples. Each graph represents the copy-number (CN) changes detected per chromosome in each sample. Top graphs correspond to LGSC patient \#6; CN changes detected in one of her recurrent tumor tissues was compared to the $\mathrm{CN}$ changes detected in the primary cell culture derived from this tissue. Bottom graphs correspond to the LGSC patient \#9; CN changes detected in one of her recurrent tumor tissues was compared to the CN changes detected in the cell line established from a later recurrent tissue. High genomic profile correlation was observed between cells and tumors in both cases

Results from the WES analysis in our LGSC cell lines and primary cultures $(n=14)$ show variable levels of genomic aberration and non-synonymous mutations (NsMs), ranging from 1 to $66 \%$ total genome change and 24-111 mutation calls per cell line (Additional file 3: Table S2a). Deletion of Chr9p, including loss of MTAP and $C D K N 2 A$ tumor suppressor genes, was found in all samples. As expected, $K R A S$ and $N R A S$ non-synonymous mutations were both found most frequently. Either mutation was present in $28.6 \%$ of all cell lines/cultures, and in $36.4 \%(K R A S)$ and $27.3 \%(N R A S)$ respectively when analyzed by patient (some cell lines were derived from the same patient at different times). KRAS and NRAS mutations co-existed in only one cell line (VOA-8862). Only one BRAF mutation was detected (D594G variant; VOA- 6800 culture). Additionally we analyzed gene mutations and copy-number changes affecting 61 wellknown MAPK-pathway genes is shown in Additional file 3: Table S2b. A summary of all RAS mutations, copynumber variation $(\mathrm{CNV})$ findings, and MEKi sensitivity in each LGSC cell culture $(n=14)$ is shown in Table 1.

We found that all MEKi-Se cell lines (4/4; iOvCa241, VOA-1312, VOA-9164, VOA-8862) carried oncogenic mutations in KRAS (G12D or G12V), while MEKi-Re cell lines were either NRAS mutant (3/7; VOA-1056/ VOA-3993 and VOA-6406), or KRAS/NRAS wt (4/7; VOA-3448/VOA-3723 and VOA-4627/VOA-4698). Of interest, the VOA-8862 cell line (mutations in both KRAS and NRAS) was found to be sensitive to all four MEKi tested. In this line, the KRAS mutation variant detected (G12D) is known to be oncogenic, while the NRAS mutation variant detected $(\mathrm{C} 118 \mathrm{Y})$ was not found in the COSMIC database, therefore its oncogenic potential remains unknown. We did not observe any obvious correlation between the degree of CNV in each cell line (copy number high versus low) and MEKi response.

\section{Proteomic differences between MEKi-Se and MEKi-Re LGSC cell lines}

To identify biomarkers of MEKi response, we compared two MEKi-Se (VOA-1312, iOvCa241) and six MEKi$\mathrm{Re}$ (VOA-1056/VOA-3993, VOA-3448/3723, VOA4627/VOA-4698) LGSC cell lines using reverse phase protein array (RPPA) analysis. To do so, lines treated with DMSO, $1 \mu \mathrm{M}$ selumetinib, or $0.1 \mu \mathrm{M}$ trametinib were screened using a panel of 91 validated antibodies (see "Materials and methods" and Additional file 1: Table S1). We found 12 proteins that were differentially expressed between MEKi-Se and MEKi-Re cell lines (Additional file 4: Table S3). Among these proteins, EGFR and PKC-alpha were found to be overexpressed in all MEKi-Re lines independently of the treatment status. These two candidates were selected for validation and further study as they are regulators of MAPK signaling and play a role in MEKi and chemotherapy resistance in the literature [33-37]. WB analysis confirmed these findings (Fig. 2a), and also showed that p-EGFR (Y1068) was overexpressed in the MEKi-Re lines. Subsequently we subsequently validated the same candidates in the three newly established LGSC cell lines (VOA-6406, VOA-8862, VOA-9164) (see Fig. 2b). In keeping with the discovery cohort results, the MEKiRe line (VOA-6406) expressed much higher levels of EGFR, p-EGFR and PKC-alpha than the two MEKi-Se lines (VOA-9164, VOA-8862) (Fig. 2b). As we previously described, $\mathrm{p}$-MEK and p-ERK1/2 expression were not found to distinguish sensitive and resistant lines by WB [24].

We subsequently assessed differential protein expression by RPPA between MEKi-Se and MEKi-Re cell lines after selumetinib and trametinib treatment. Twentyone and seventeen proteins were significantly different between MEKi-Se and MEKi-Re cells after selumetinib and trametinib treatment, respectively (Additional file 4: Table S3). Confirmation of the RPPA results was assessed by WB in one representative cell line from each individual patient (VOA-3993, VOA-4627, VOA-3723). Cell lines derived from the same patients at different time points in the disease course were not included for this analysis (VOA-1056, VOA-4698, VOA-3448). As seen in the untreated cells, WB confirmed increased p-EGFR levels in the MEKi-Re cell lines (Fig. 2c, d). As expected, trametinib more effectively inhibited MAPK phosphorylation than selumetinib. Differences in GSK3B and BID protein expression were also observed between MEKi-Se versus MEKi-Re cells by RPPA, however we were unable to validate these results using mass spectrometry (MS) analysis (data not shown). Interestingly, a number of the differentially expressed proteins (MEK-Se versus MEK$\mathrm{Re})$ were found to be drug-specific. These RPPA screening results are summarized in Additional file 4: Table S3, though these findings require further validation. 


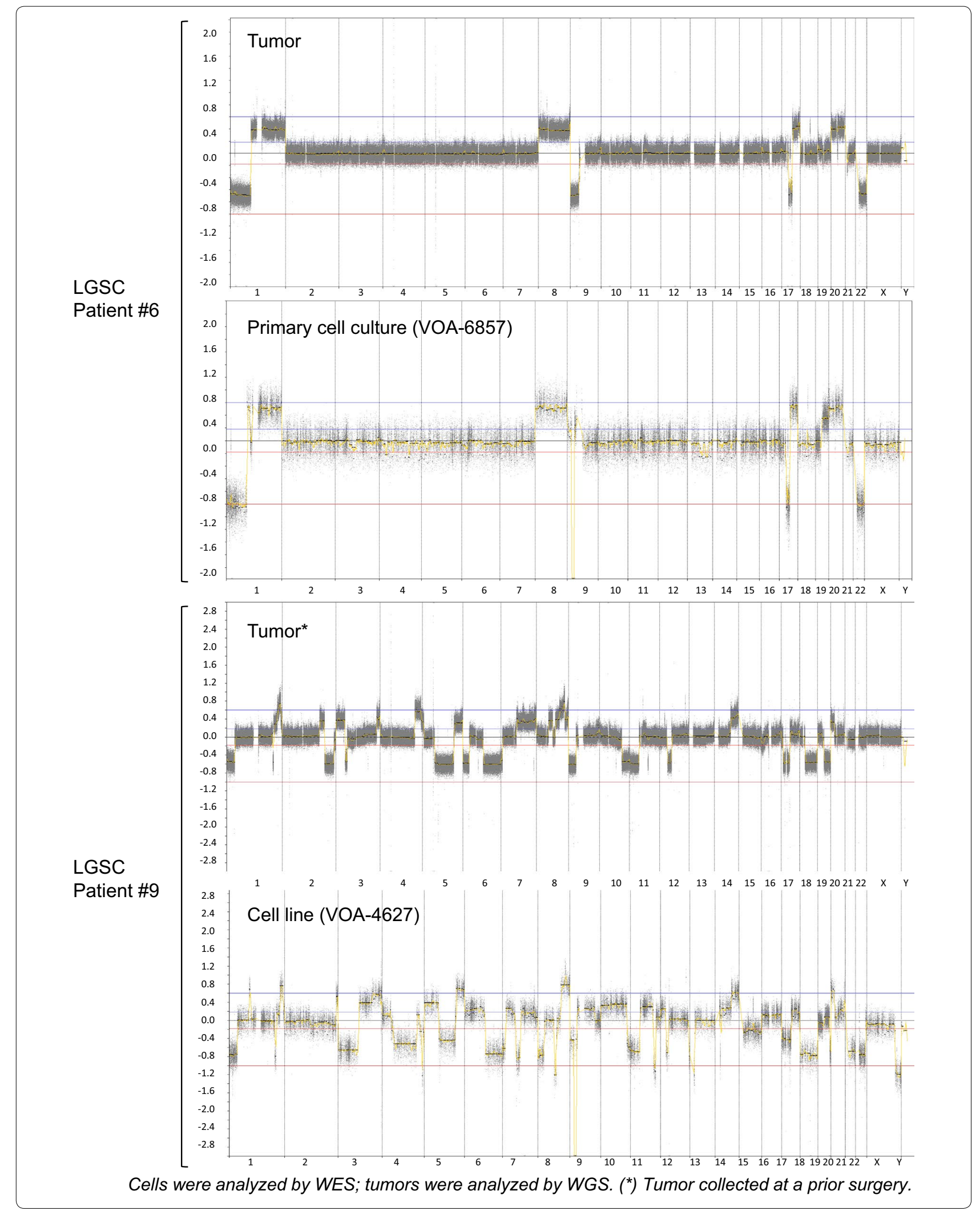




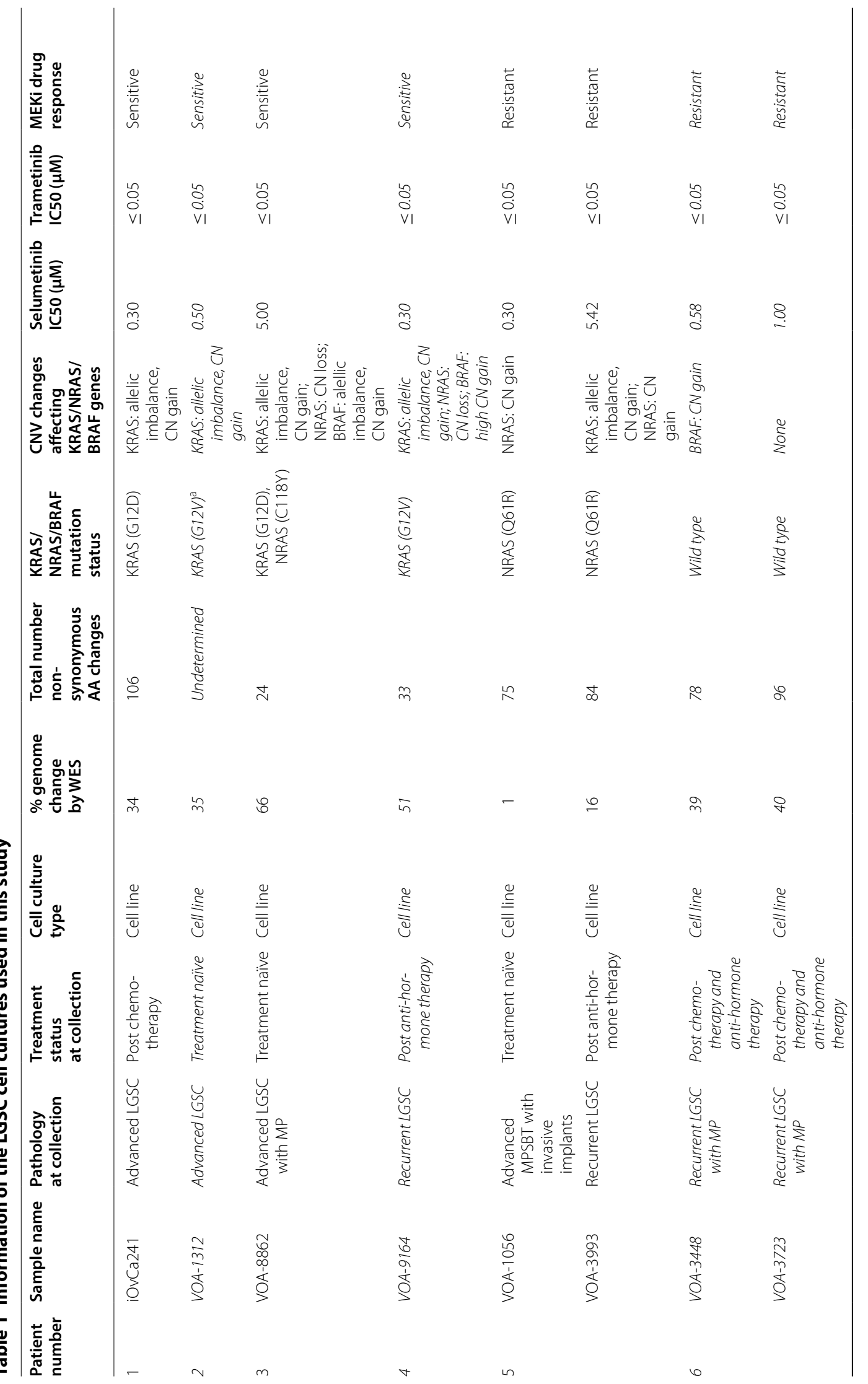




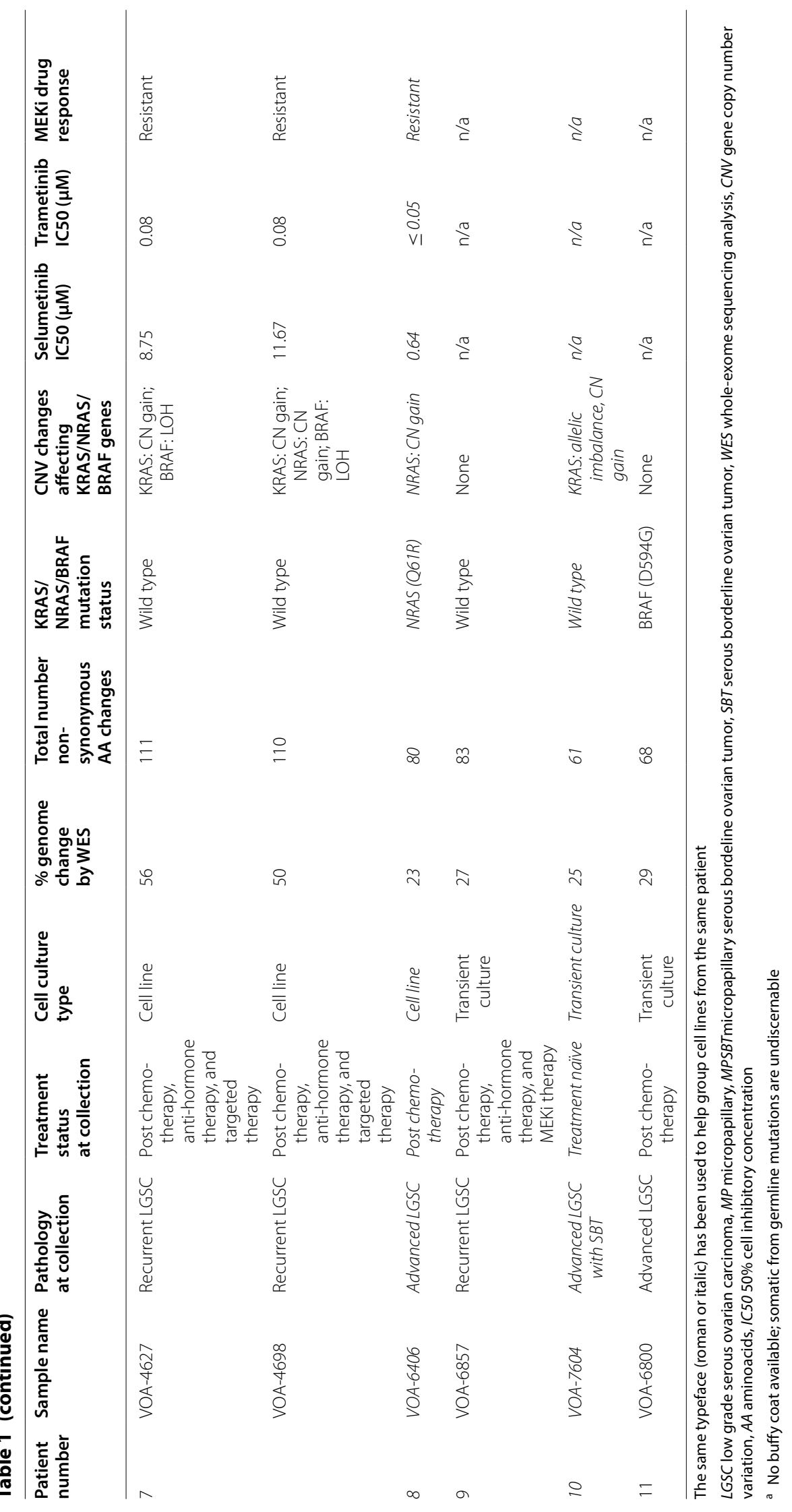



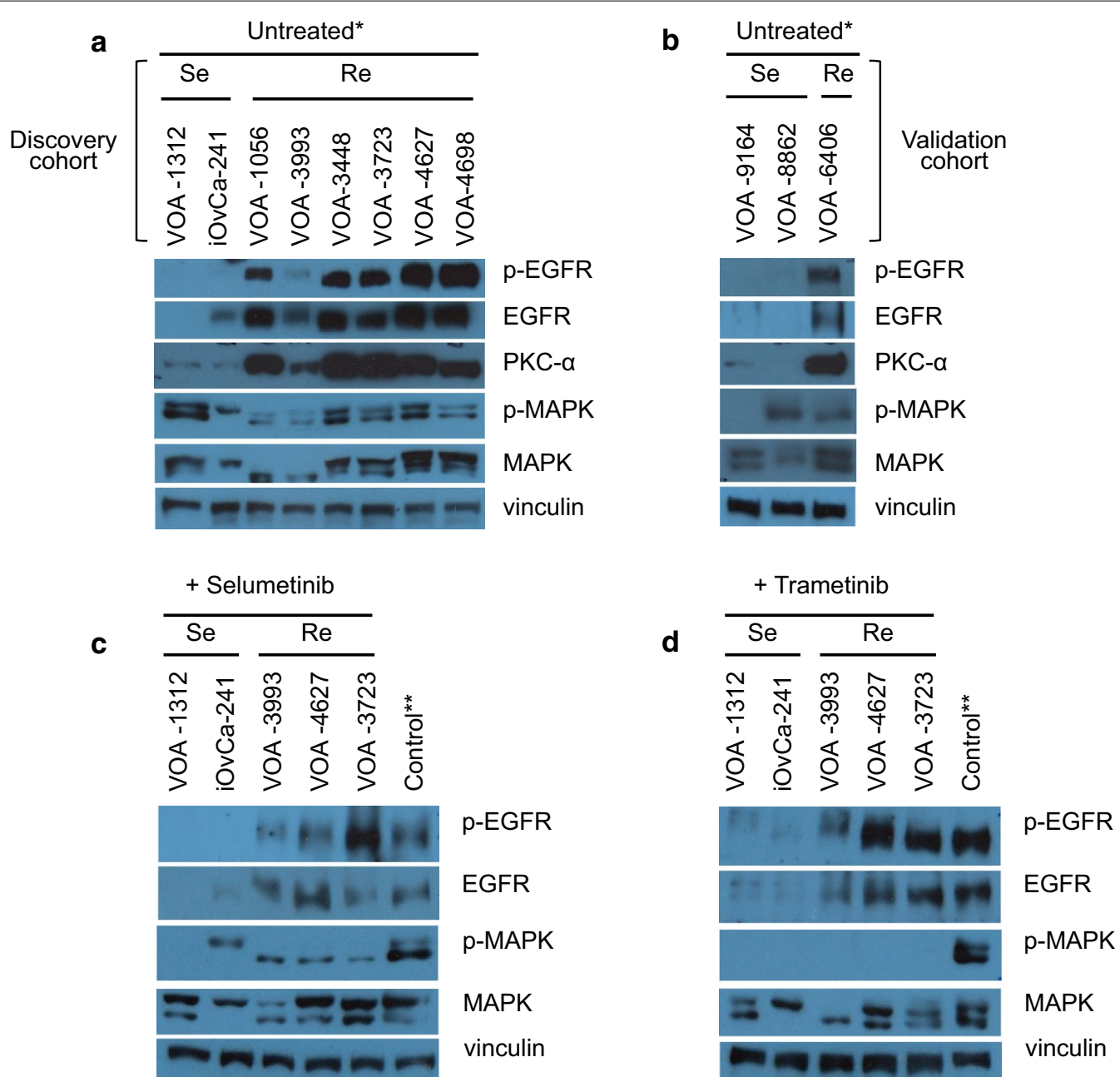

Fig. 2 Differential expression of EGFR, p-EGFR and PKC-a between MEKi-Se and MEKi-Re LGSC cell lines by WB. a Confirmation of the RPPA results in untreated MEKi-Se and MEKi-Re lines (a, discovery cohort). EGFR, p-EGFR and PKC-a were increased in MEKi-Re lines $(n=5)$ compared to MEKi-Se lines $(n=2)$. $\mathbf{b}$ Validation of these protein biomarkers in three newly established LGSC cell lines classified according to their MEKi responsiveness (validation cohort). As found in the cell lines analyzed by RPPA, the new MEKi-Re line $(n=1)$ expressed higher levels of EGFR, $p$-EGFR and PKC-a compared to the two new MEKi-Se lines tested $(n=2)$. $\mathbf{c}$, $\mathbf{d}$ Confirmation of RPPA results in MEKi treated cell lines. With MEKi treatment (selumetinib $1 \mu \mathrm{M}$ and trametinib $0.1 \mu \mathrm{M})$ p-EGFR expression remained higher in MEKi-Re lines. As previously described, trametinib showed stronger inhibitory effects on MAPK (p-MAPK or p-ERK1/2) than selumetinib, even when used at ten times lower dose. $\left(^{*}\right)$ No DMSO. $\left(^{* *}\right)$ Untreated VOA-4627 cells to control for drug inhibition effects on MAPK pathway

\section{In vitro evaluation of MEK and EGFR inhibition in MEKi-Re LGSC cell lines}

To establish whether EGFR expression played a role in mediating MEKi resistance we evaluated the effects of EGFR inhibition (using erlotinib), with and without MEK inhibition (using selumetinib or trametinib), in four MEKi-Re LGSC cell lines (VOA-3723, VOA-3993, VOA4627, and VOA-6406). IC50 values for erlotinib in these cell lines are shown in Additional file 5: Table S4. Except for VOA-3723, all MEKi-Re lines were highly resistant to single erlotinib treatment as observed in other ovarian cancer cell lines [38, 39]. Erlotinib doses chosen for the combined experiments are in keeping with erlotinib human serum levels [40]. Effects of single and combined drug treatment were evaluated using proliferation, viability, and WB assays. EGFR mutation and copy-number status were also evaluated. By WES, none of our LGSC cell lines carried activating mutations in EGFR, though some had copy-number changes affecting this gene. As summarized in Additional file 5: Table S4, we could not identify any obvious factors [EGFR CNV levels, levels of EGFR protein expression, phosphorylation, or sensitivity (IC50 values) to erlotinib treatment] that were associated with sensitivity to combination therapy. 

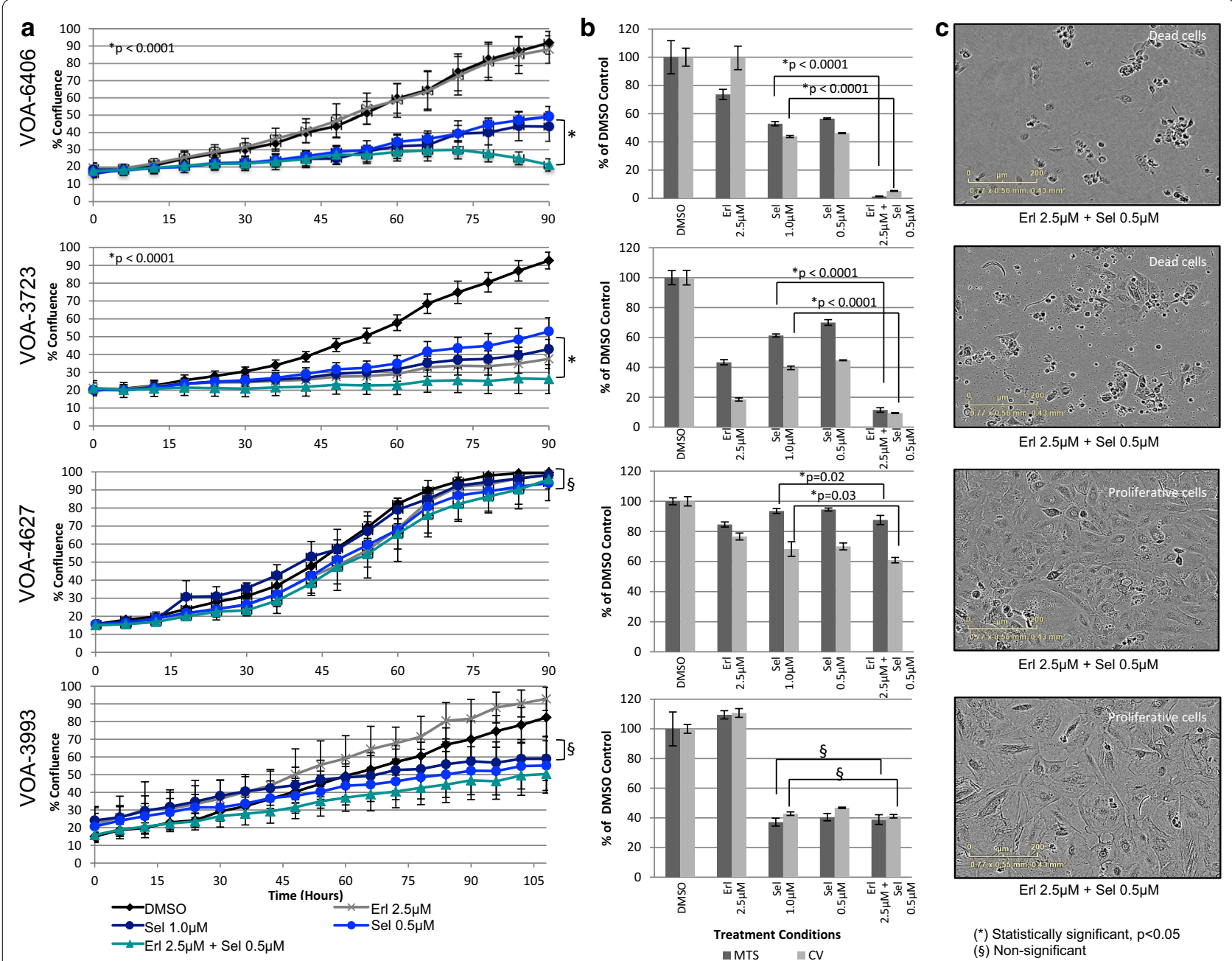

Fig. 3 Effects of selumetinib and erlotinib single and combined drug treatments in four MEKi-Re LGSC cell lines. The graphed curves (a) represent the results from the proliferation experiments and the bar graphs (b) represent the results from the viability (MTS and CV) assays performed at the end of the proliferation experiments. The photomicrographs (c) show IncuCyte ${ }^{\mathrm{TM}}$ images at the completion of the experiment. All four cell lines were resistant to single selumetinib and erlotinib treatments. However, when the drugs were combined, VOA-6406 and VOA-3723 cell lines demonstrated complete cell death while VOA-4627 and VOA-3993 cells were shown to be resistant to the dual selumetinib and erlotinib treatment combination

With the highest dose of erlotinib treatment alone ( $2.5 \mu \mathrm{M}$, one dose, over 4-5 days), all four MEKi-Re cell lines continue to proliferate. Interestingly as shown in Fig. 3, a reduced dose of selumetinib $(0.5 \mu \mathrm{M})$ in combination with erlotinib $(2.5 \mu \mathrm{M})$ resulted in statistically significant decreases in cell proliferation and viability $(\mathrm{p}<0.001$; $\mathrm{t}$-test) in 2 of the 4 cell lines tested (VOA-3723 and VOA-6406). At the end of these experiments, complete cell death of both cell lines was confirmed by image inspection on Incucyte $^{\mathrm{TM}}$ (Fig. 3; and Additional file 6: Figure S2). Using Compusyn software analysis, synergistic drug effects (were demonstrable even using lower doses of erlotinib $(1.25$ or $0.63 \mu \mathrm{M})$ with selumetinib
$(0.5 \mu \mathrm{M})$ (Additional file 7: Table S5). These drug combinations were not effective in the other two lines tested (VOA-3993 and VOA-4627). Reduced dose of trametinib $(0.05 \mu \mathrm{M})$ in combination with erlotinib $(2.5 \mu \mathrm{M})$ resulted in similar results for the VOA-3723 cell line, but cytostatic effects for the VOA-6406 cell line. A summary of the synergistic drug effects are shown in Additional file 7: Table S5. Drug synergy was stronger with selumetinib and erlotinib combination than with trametinib and erlotinib combination.

The effects of erlotinib, with and without MEKi treatment, on EGFR and MAPK signaling pathways were evaluated using WB. Levels of total and phosphorylated 
EGFR and ERK1/2, as well as total and cleaved PARP (c-PARP) were measured after $24 \mathrm{~h}$ treatment. Results from these experiments indicated that drug effects on cell signaling were cell line dependent (Fig. 4; and Additional file 8: Figure S3). As previously reported by our group, trametinib alone $(0.1 \mu \mathrm{M})$ caused stronger inhibitory effects on ERK1/2 phosphorylation (p-ERK1/2) than selumetinib $(1 \mu \mathrm{M})$. Unexpectedly, selumetinib treatment increased EGFR phosphorylation (p-EGFR Y1068) in 3 out of 4 MEKi-Re cell lines (VOA-6406, VOA-3723, and VOA-4627), however these effects were less obvious with trametinib treatment. As expected, erlotinib alone inhibited EGFR phosphorylation (p-EGFR Y1068) in all cell lines. Interestingly, erlotinib alone also inhibited ERK1/2 phosphorylation in 2 out of 4 lines (VOA-3723 and VOA4627) and activated ERK1/2 phosphorylation in another line (VOA-6406). No pathway interaction was detected in the resistant VOA-3993 cell line. In these lines, while pathway interaction was observed, none of the changes in p-EGFR Y1068, p-ERK1/2 or c-PARP correlated with sensitivity or resistance to dual EGFRi and MEKi treatment. In the two MEKi-Re lines resistant to combination therapy (MEKi and erlotinib), the trametinib and erlotinib combination resulted in more apoptosis induction than the selumetinib and erlotinib combination (as measured by c-PARP).

\section{Effects of PKC-alpha inhibition in MEKi-Re LGSC cell lines} Genomic characterization of PRKCA by WES revealed that none of our LGSC cell lines carried activating mutations in PRKCA. It is interesting to note that two MEKiRe cell cultures (VOA-3723 and VOA-6857) carried PRKCA copy-number gain and two MEKi-Se cells (VOA9164 and VOA-8862) had PRKCA copy-number loss. To determine whether $\mathrm{PKC}$-alpha protein expression played a role in mediating MEKi resistance we evaluated the effects of PKC-alpha knockdown using lentiviral shRNA, with and without selumetinib or trametinib in two MEKi-Re LGSC cell lines (VOA-6406 and VOA-3723). As shown in Fig. 5a, PRKCA shRNA resulted in a complete PKC-alpha protein knockdown in VOA-6406 cells and a partial knockdown in VOA-3723 cells by WB. Subsequent MEKi treatment $(1.0 \mu \mathrm{M}$ selumetinib or $0.1 \mu \mathrm{M}$ trametinib) experiments showed no significant changes

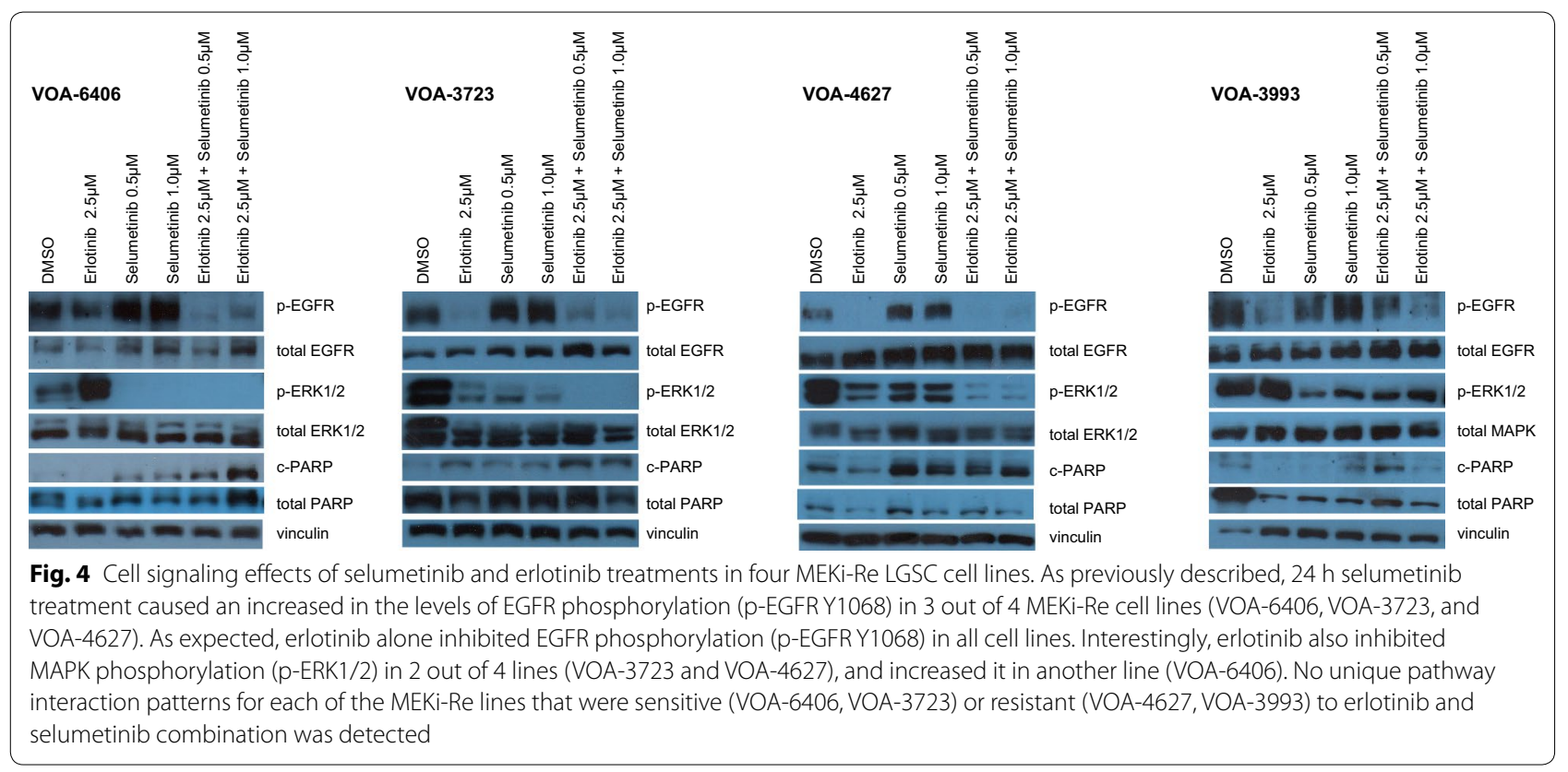

(See figure on next page.)

Fig. 5 Effects of PRKCA knockdown in two MEKi-Re LGSC cell lines using lentiviral shRNA. a Determination of PKC-alpha (PKC-a) protein expression by WB. Transduction with lentiviral particles containing PRKCA shRNA resulted in a complete PKC-a protein knockdown in VOA-6406 cells and a partial knockdown in VOA-3723 cells. As detected by WES, VOA-3723 cells display PRCKA CN gain, which may explain the partial PKC-a protein knockdown. $\mathbf{b}$ Effects of PRKCA knockdown on cell proliferation. Reduction of PKC-a levels alone did not compromise cell proliferation in either of the two cell lines tested. Furthermore, PRKCA knockdown in combination with selumetinib treatment did not seem to significantly increase the sensitivity of these lines to selumetinib treatment. c Effects of PRKCA knockdown on cell viability (MTS and CV assays). As seen in these bar graphs, and similar to what we observed in the proliferation experiments, PRKCA knockdown did not seem to impact the viability of these lines 


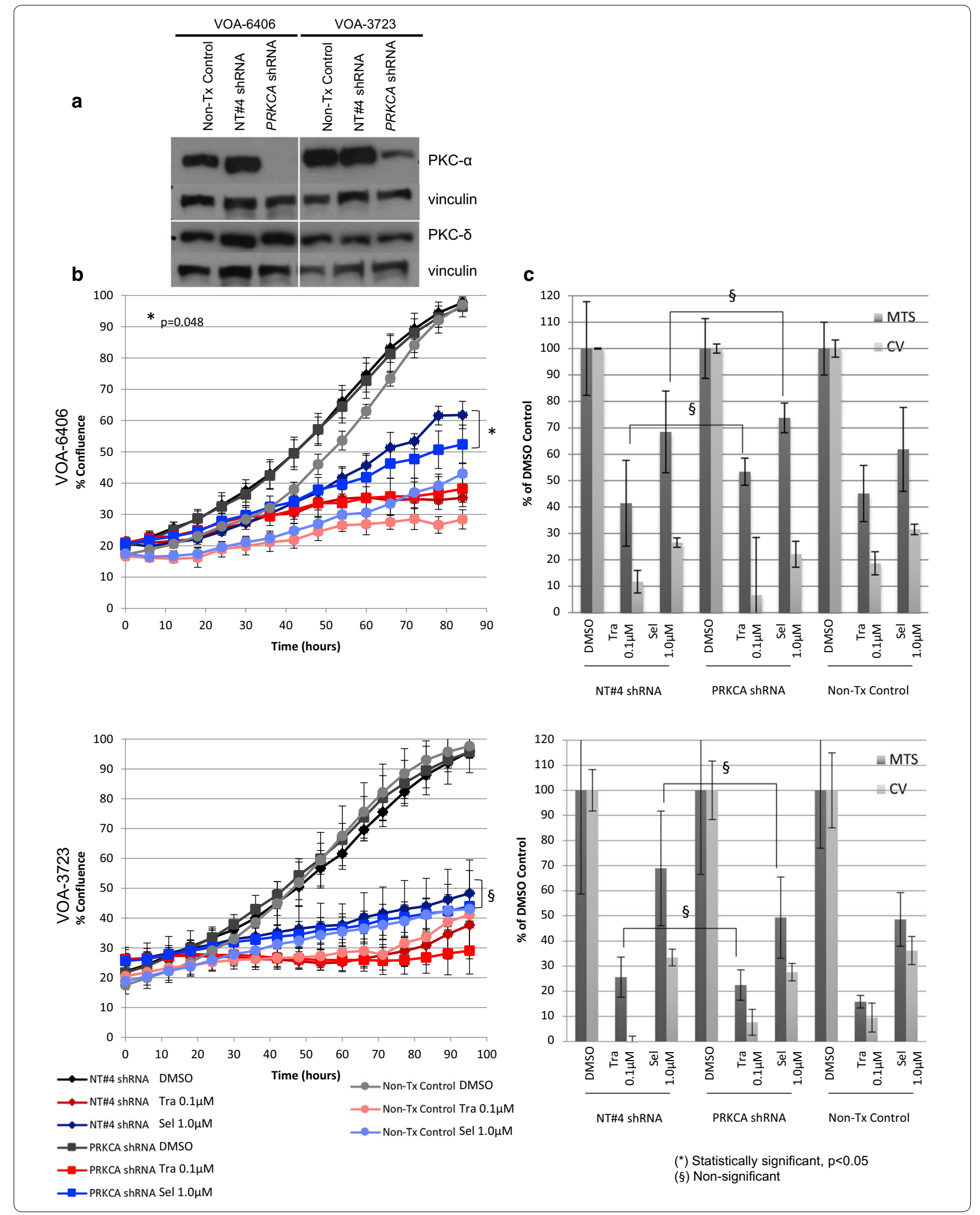


in cell viability when compared to non-target shRNA transduced lines (Fig. 5b). Proliferation assays demonstrated that PKC-alpha knockdown did not increase MEKi sensitivity in the VOA-3723 cells, but may slightly increase MEKi sensitivity to selumetinib in the VOA6406 cell line $(p=0.048)$. This treatment combination was much less effective than EGFRi and MEKi combination in MEKi-Re LGSC cell lines as the cells continue to proliferate. Taken together, these results do not support PKC-alpha as a treatment target in LGSC to expand the efficacy of MEKi treatment.

\section{Discussion}

Activating mutations affecting the MAPK pathway (RAS/ RAF/MEK/ERK) are frequently found in cancer. MAPK pathway inhibitors, such as MEK inhibitors, were developed as targeted therapeutics to potentially treat such cancers [41, 42]. MEKi as single agents or in combination with other therapies have been studied for the treatment of melanoma, lung and colorectal cancers [43]. In 2013, the MEKi selumetinib was evaluated in a phase II clinical trial as a treatment for LGSC. Clinical responses (RECIST-1.1) to MEKi were observed in 15\% of patients $[22,44]$. While these responses were limited, response rates using conventional chemotherapy in patients with relapsed LGSC are disappointingly low (4\%) [45]. More recently, a number of LGSC cases have been reported, highlighting dramatic and durable responses to MEKi treatment $[22,23,46,47]$. Currently, there are no predictive biomarkers of MEKi response for LGSC. Identifying molecular markers which predict MEKi treatment efficacy will allow for pre-selection of patients who would benefit from this treatment, and avoid ineffective treatments and toxicities in those patients unlikely to respond.

In this study, we utilized genomic and proteomic techniques to molecularly characterize a collection of LGSC cell lines and primary cultures (derived from advanced/ recurrent LGSC patients), and identify markers that predict response (sensitivity/resistance) to MEKi treatment in vitro. Genomic profiles of two of these cell models were compared with their corresponding tumor samples from the same patient and showed remarkably similar copy number profiles, supporting the utility of these cell models for preclinical research. Subsequent comparisons of genomic profiles from an additional twelve LGSC cell models showed frequent deletion of Chr9p (including loss of MTAP and CDKN2A genes) [48, 49] and oncogenic mutations in KRAS and NRAS genes, in agreement with results from previous studies on LGSC tumor tissues [13-15]. Additionally, RAS mutations were often associated with $R A S$ copy number gain. As previously reported $[24,46,50]$ we also detected multiple and distinct genomic alterations affecting other genes related to the MAPK cell signaling pathway. It is worth noting that the individual comparison of genomic profiles between LGSC cultures showed substantial variations in the types of gene mutations and copy-number alterations, indicating widespread molecular differences in LGSC tumors between patients.

Further evaluation of mutation profiles in eight LGSC cell lines with different sensitivity to MEKi treatment (two MEKi-Se and six MEKi-Re) showed oncogenic mutations in KRAS in all four MEKi-Se lines which were absent in all six MEK-Re lines. Previous results from a clinical trial using selumetinib (Farley et al. [22]) did not find a significant relationship between $R A S$ mutation status and MEKi response rates in LGSC patients. It is important to note that tumor samples were not available for testing in $35 \%$ of the patients (18 of 52) in this study. In agreement with our results, two recent case reports on LGSC patients with remarkable and durable clinical responses ( $>5$ years) to MEKi therapy have reported oncogenic KRAS mutations (both $\mathrm{G} 12 \mathrm{~V}$ ) in their tumors $[23,47]$. As LGSC is often an indolent disease, the inclusion of patients with stable disease should also be considered in the future evaluation of RAS mutation status as a predictive biomarker. It is not unexpected that a single biomarker, such as KRAS mutation status, will not accurately predict responses to MEKi treatment, recognizing that LGSC harbor other MAPK-pathway gene mutations and significant MAPK copy number changes. Furthermore, KRAS copy-number amplification (described as one activating mechanism) could also play a role in mediating MEKi efficacy [44].

Using RPPA to compare MEKi-Se and MEKi-Re LGSC cell lines, we found that all MEKi-Re lines had higher levels of EGFR and PKC-alpha expression. These results were subsequently validated in three newly established LGSC cell lines. Using this approach, we also described proteomic changes specific to each MEKi tested (selumetinib or trametinib). The changes we observed may be particularly relevant when evaluating differences in drug efficacy, as MEKi may exhibit differences in MEK isoform specificity or off-target effects [24]. Interestingly, all MEKi-Re lines expressed higher levels of EGFR activation (p-EGFR Y1068) than the MEKi-Se lines. Although our study was limited to a small number of cell lines, we did not observe an obvious correlation between levels of EGFR and PKC- $\alpha$ protein expression and specific gene mutations or copy number changes in these genes.

In colorectal cancer, preclinical studies with BRAF inhibitors have reported adaptive feedback reactivation of MAPK signaling involving EGFR [33, 51]. This feedback signaling can be blocked by the addition of a MEKi. We similarly found evidence of MAPK feedback signaling following MEKi treatment that appears to play a role 
in MEKi resistances. Half of the MEKi-Re cells (2/4 cell lines) were effectively treated with selumetinib in combination with erlotinib, causing complete cell death. Combination therapy was effective in these two cell lines using drug doses that were below those that lacked efficacy as single drug treatments. Drug synergy was demonstrated using CompuSyn analyses in the two cell lines where cell death was demonstrated. In contrast, the other two lines tested continued to proliferate even with higher doses of the drug combination. We were unable to observe any obvious changes in p-EGFR and/or p-ERK that characterized the two combination-therapy resistant cell lines. As seen in our previous study [24], trametinib appeared to be a more effective inhibitor of ERK phosphorylation and cell proliferation than selumetinib. Based on its enhanced efficacy, it was more difficult to detect drug synergism using the erlotinib/trametinib combination than with the erlotinib/selumetinib combination.

There is a growing body of evidence supporting the use of combining a targeted therapy with other targeted agents or with traditional chemotherapeutic agents [29, 52]. Combination therapy using erlotinib and selumetinib was studied in a randomized phase II trial in lung cancer [53]. This drug combination did not prove to be effective in lung cancers irrespective of KRAS mutant status. Though the treatment was tolerated, significant side effects occurred with combination therapy. If these drug treatment combinations are going to be effective in LGSC, optimal drug dosing will be required in order to minimize side effects without loss of treatment efficacy.

Combination therapy with BRAFi and MEKi has remarkably improved survival in the adjuvant setting for patients with $B R A F$ mutant melanomas, and combining a BRAFi and an EGFRi has improved tumor regression in $B R A F$ mutant colorectal cancer xenografts $[51,54]$. In a recent report, binimetinib in combination with paclitaxel was studied in platinum resistant ovarian cancer patients (NCT01649336). Two LGSC patients included in this trial showed response to this drug combination. These cases had also the largest reduction in target lesion size among the 25 ovarian cancer patients studied. MAPK pathway aberrations (KRAS G12D mutation and a CUL1:BRAF fusion) were identified in the tumors of both patients [44]. Additionally, two more LGSC patients with KRAS G12V [23, 47] and one with MEK1 (Q56_V60del) gene mutations experienced disease stabilization in response to this drug treatment combination [46].

PKC-alpha expression has been implicated in chemotherapy drug resistance in some cancers [36, 37]. To explore its potential role in MEKi resistance, we inhibited PKC- $\alpha$ expression in two MEKi-Re lines. In the cell line where complete $\mathrm{PKC}$-alpha protein knockdown was achieved, the effect of this treatment combination was not nearly as effective as combining MEKi and EGFRi. In the other line, where only partial knockdown of PKC-alpha protein expression was obtained, no changes in MEKi sensitivity were observed. Of interest, we found that this line contained PRKCA copy number gain. PKC-alpha knockdown by itself did not affect cell proliferation in either cell line. The results of our experiments suggest that $\mathrm{PKC}$-alpha protein expression appears to be a predictive biomarker but is not a therapeutic target mediating MEKi resistance.

Identifying molecular characteristics to predict drug sensitivity/resistance in individual patients with solid tumors has proved to be challenging. The efficacy of therapies designed to target specific mutations are known to be dependent on the cancer type. For example, while BRAF inhibitors have shown to be effective in melanomas carrying BRAF mutations, they have demonstrated little effect in the treatment of $B R A F$ mutant colon cancers $[33,55,56]$. In advanced LGSC, mutations in KRAS are more common than in $B R A F[14,19,57,58]$. While MEKi have shown efficacy in some LGSC, still only a minority of patients respond to this treatment. Thus, it is of utmost importance to identify markers of drug treatment efficacy specific for each cancer type. A current clinical trial using the MEKi trametinib to treat patients with LGSC (NCT02101788), will include a translational research component in an attempt to identify predictive biomarkers in patient tumor samples.

\section{Conclusions}

In summary, this proteogenomic study is the first to perform predictive biomarker discovery for MEKi treatment in LGSC cell lines. MEKi-Se cell lines were found to have oncogenic KRAS mutations and low levels of EGFR and PKC-alpha protein expression. The confirmation of these results in MEKi treated LGSC tumors samples could lead to better patient selection for MEKi treatment, and further avoid unnecessary treatment and toxicities in patients unlikely to respond. Our study also suggests that a significant portion of those LGSC patients whose tumors are unresponsive to MEKi therapy may benefit from combination therapy with EGFR and MEK inhibition. As LGSC xenografts are not yet available for research, we are currently unable to validate these results in vivo. However, we are now using our LGSC patient-derived cell lines to establish xenograft models. The potential predictive value of the three molecular markers of MEKi response identified in our LGSC cell line models should be considered 
for further validation in clinical trials using MEKi for the treatment of LGSC.

\section{Additional files}

\section{Additional file 1: Table S1. List of antibodies included in the RPPA} analysis.

Additional file 2: Figure S1. Preclinical MEKi evaluation in novel LGSC cell lines.

Additional file 3: Table S2. List of all NsMs and genomic aberrations (CNV and NsMs) affecting 61 MAPK-related genes (WES analysis).

Additional file 4: Table S3. List of proteins differentially expressed between MEKi-se and MEKi-re LGSC cell lines (results from the RPPA and SPSS analysis).

Additional file 5: Table S4. EGFR information on LGSC cell lines.

Additional file 6: Figure S2. Biological effects of trametinib and erlotinib drug combination in MEKi-re LGSC cell lines.

Additional file 7: Table S5. Compusyn data from selumetinib/trametinib and erlotinib drug combinations in MEKi-re LGSC cell lines.

Additional file 8: Figure S3. Effects of trametinib and erlotinib combination on LGSC cell lines by WB analysis.

\section{Abbreviations}

BCCA: British Columbia Cancer Agency; BCCF: British Columbia Cancer Foundation; C-PARP: cleaved PARP; Chr: chromosome; Cl: combination index; CNA: copy-number aberration; CNV: copy-number variation; CV: crystal violet; dbSNP: single nucleotide polymorphism database; dFBS: defined fetal bovine serum; DMSO: dimethylsulfoxide; EGFRi: EGFR inhibitor; Erlo: erlotinib; GOC: Society of Gynecologic Oncology of Canada; HGSC: high-grade serous ovarian cancer/carcinoma; HRP: horse radish peroxidase; LGSC: low-grade serous ovarian cancer/carcinoma; MEKi-re: MEK inhibitor resistant; MEKi-se: MEK inhibitor sensitive; MEKi: MEK inhibitor; MTS: tetrazolium compound 3-(4,5-dimethylthiazol-2-yl)-5-(3-carboxymethoxyphenyl)-2-(4-sulfophenyl)-2H-tetrazolium; NsMs: non-synonymous mutation; OvCaRe: British Columbia Ovarian Cancer Research Program; p-EGFR: phosphorylated EGFR; p-ERK: phosphorylated ERK; p-MEK: phosphorylated MEK; PBS: phosphate saline buffer; PDX: patientderived xenografts; PKC-a: PKC-alpha; POG: Personalized Oncogenomics Program; RPPA: reverse phase protein array; Sel: selumetinib; shRNA: short hairpin RNA; SNP: single nucleotide polymorphism; STR: short tandem repeat; Tra: trametinib; UBC: University of British Columbia; WB: western blot; WES: whole exome sequencing; WGS: whole genome sequencing; WB: western blot; p-val: p-value.

\section{Authors' contributions}

MLF performed research conception, design, experimental preparation, data analysis, data interpretation, and manuscript preparation; AD performed experimental preparation, data analysis, and manuscript preparation; JH performed western blot, cell proliferation and viability, drug testing and data analysis; HK performed western blot preparation and data analysis, and clinical data collection; SB performed RPPA experimental sample preparation and data analysis; CS assisted with cell culture work and cell line generation; GD assisted with cell line generation, data interpretation and manuscript revision; TS assisted with cell line generation; MC performed RPPA experimental work and data analysis; BH lead the RPPA experimental work and data analysis; SA performed CNV analysis and figure preparation; SV performed mutation call analysis; CCC assisted with genomic analysis and data interpretation; DGH supervised the conduct of the research, acquisition of data and banked tumor samples; MSC lead this research, developed the research concepts, statistical data analysis, data interpretation, and manuscript preparation and review. All authors read and approved the final manuscript.

\section{Author details}

${ }^{1}$ Obstetrics and Gynecology, University of British Columbia, Vancouver, BC, Canada. ${ }^{2}$ Pathology and Laboratory Medicine, University of British Columbia, Vancouver, BC, Canada. ${ }^{3}$ Translational Ovarian Cancer Research Program, London Health Science Centre, London, ON, Canada. ${ }^{4}$ Oncology, University of Western Ontario, London, ON, Canada. ${ }^{5}$ Medical Oncology, Royal College of Surgeons in Ireland, Beaumont Hospital, Dublin, Ireland. ${ }^{6}$ Laboratory for Advanced Genome Analysis, Vancouver Prostate Centre, Vancouver, BC, Canada. ${ }^{7}$ Molecular Oncology, British Columbia Cancer Agency, Vancouver, BC, Canada. ${ }^{8}$ Division of Gynecologic Oncology, Diamond Health Centre, 2775 Laurel St., 6th Floor, Vancouver, BC V5Z 1M9, Canada.

\section{Acknowledgements}

This work was supported by the British Columbia Cancer Foundation (BCCF) and the British Columbia Ovarian Cancer Research (OvCaRe) Program. The authors want to extend a special thanks to the MacKenzie and Lawler families, the Janet D. Cottrelle Foundation, and to all patients, families, and donors who supported this research. We would also like to thank the Society of Gynecologic Oncology of Canada (GOC) for their support in advancing research and knowledge translation in LGSC.

\section{Competing interests}

The authors declare that they have no competing interests.

\section{Availability of data and materials}

Most of the relevant data generated or analysed for this study is included in this published article and/or additional files. Additional data is available from the corresponding author on reasonable request.

\section{Consent for publication}

This manuscript does not contain any identifiable patient data.

\section{Ethics approval and consent to participate}

The research relating to this study was approved by the institutional human ethics review boards at BCCA (H14-02859), the University of British Columbia (R05-0119), and the University of Western Ontario (HSREB 12668E). Patient consent was required and obtained for tumor banked samples.

\section{Funding}

Dr. Mark S. Carey and authors affiliated with the University of British Columbia are supported by funds from the British Columbia Cancer Foundation (BCCF), Vancouver General Hospital Foundation and a grants from Cancer Research Society. Dr. T. Shepherd is supported by a grant from the Canadian Institute of Health Research Grant \#136836. Dr. Huntsman and authors affiliated with the Department of Pathology and Laboratory Medicine are supported by funds from the BCCF, the University of British Columbia (UBC) Hospital Foundation and grants from the Canadian Institute of Health Research (CIHR), Canada Research Society.

\section{Publisher's Note}

Springer Nature remains neutral with regard to jurisdictional claims in published maps and institutional affiliations.

Received: 19 October 2018 Accepted: 2 January 2019

Published online: 08 January 2019

\section{References}

1. Society AC. Cancer facts and figures 2017.

2. Society CC. Canadian cancer statistics 2017.

3. Matsuo K, Machida H, Grubbs BH, Sood AK, Gershenson DM. Trends of low-grade serous ovarian carcinoma in the United States. J Gynecol Oncol. 2018:29:e15.

4. Gourley C, Farley J, Provencher DM, Pignata S, Mileshkin L, Harter P, Maenpaa J, Kim JW, Pujaide-Lauraine E, Glasspool RM, Ray-Coquard I, Gershenson D. Gynecologic Cancer InterGroup (GCIG) consensus review for ovarian and primary peritoneal low-grade serous carcinomas. Int J Gynecol Cancer. 2014;24:S9-13. 
5. Grabowski JP, Harter P, Heitz F, Pujade-Lauraine E, Reuss A, Kristensen G, Ray-Coquard I, Heitz J, Traut A, Pfisterer J, du Bois A. Operability and chemotherapy responsiveness in advanced low-grade serous ovarian cancer. An analysis of the AGO Study Group metadatabase. Gynecol Oncol. 2016;140:457-62.

6. Gershenson DM. Low-grade serous carcinoma of the ovary or peritoneum. Ann Oncol. 2016;27(Suppl 1):i45-9.

7. Kobel M, Kalloger SE, Boyd N, McKinney S, Mehl E, Palmer C, Leung S, Bowen NJ, lonescu DN, Rajput A, Prentice LM, Miller D, Santos J, Swenerton K, Gilks CB, Huntsman D. Ovarian carcinoma subtypes are different diseases: implications for biomarker studies. PLoS Med. 2008;5:e232.

8. Kurman RJ, Shih le M. Molecular pathogenesis and extraovarian origin of epithelial ovarian cancer-shifting the paradigm. Hum Pathol. 2011;42:918-31.

9. Singer G, Stohr R, Cope L, Dehari R, Hartmann A, Cao DF, Wang TL, Kurman RJ, Shih le M. Patterns of p53 mutations separate ovarian serous borderline tumors and low- and high-grade carcinomas and provide support for a new model of ovarian carcinogenesis: a mutational analysis with immunohistochemical correlation. Am J Surg Pathol. 2005;29:218-24.

10. Cancer Genome Atlas Research N. Integrated genomic analyses of ovarian carcinoma. Nature. 2011;474:609-15.

11. Wong KK, Lu KH, Malpica A, Bodurka DC, Shvartsman HS, Schmandt RE, Thornton AD, Deavers MT, Silva EG, Gershenson DM. Significantly greater expression of ER, PR, and ECAD in advanced-stage low-grade ovarian serous carcinoma as revealed by immunohistochemical analysis. Int J Gynecol Pathol. 2007;26:404-9.

12. Ayhan A, Kurman RJ, Yemelyanova A, Vang R, Logani S, Seidman JD, Shih le M. Defining the cut point between low-grade and high-grade ovarian serous carcinomas: a clinicopathologic and molecular genetic analysis. Am J Surg Pathol. 2009;33:1220-4.

13. Singer G, Oldt R 3rd, Cohen Y, Wang BG, Sidransky D, Kurman RJ, Shih le M. Mutations in BRAF and KRAS characterize the development of lowgrade ovarian serous carcinoma. J Natl Cancer Inst. 2003;95:484-6.

14. Tsang YT, Deavers MT, Sun CC, Kwan SY, Kuo E, Malpica A, Mok SC, Gershenson DM, Wong KK. KRAS (but not BRAF) mutations in ovarian serous borderline tumour are associated with recurrent low-grade serous carcinoma. J Pathol. 2013;231:449-56.

15. Jones $S$, Wang TL, Kurman RJ, Nakayama K, Velculescu VE, Vogelstein $B$, Kinzler KW, Papadopoulos N, Shih le M. Low-grade serous carcinomas of the ovary contain very few point mutations. J Pathol. 2012;226:413-20.

16. Malpica A, Wong KK. The molecular pathology of ovarian serous borderline tumors. Ann Oncol. 2016:27(Suppl 1):i16-9.

17. Grisham RN, lyer G. Low-grade serous ovarian cancer: current treatment paradigms and future directions. Curr Treat Options Oncol. 2018;19:54.

18. Emmanuel C, Chiew YE, George J, Etemadmoghadam D, Anglesio MS, Sharma R, Russell P, Kennedy C, Fereday S, Hung J, Galletta L, Hogg R, Wain GV, Brand A, Balleine R, MacConaill L, Palescandolo E, Hunter SM, Campbell I, Dobrovic A, Wong SQ, Do H, Clarke CL, Harnett PR, Bowtell DD, deFazio A, Australian Ovarian Cancer S. Genomic classification of serous ovarian cancer with adjacent borderline differentiates RAS pathway and TP53-mutant tumors and identifies NRAS as an oncogenic driver. Clin Cancer Res. 2014;20:6618-30.

19. Hunter SM, Anglesio MS, Ryland GL, Sharma R, Chiew YE, Rowley SM, Doyle MA, Li J, Gilks CB, Moss P, Allan PE, Stephens AN, Huntsman DG, deFazio A, Bowtell DD, Australian Ovarian Cancer Study G, Gorringe KL, Campbell IG. Molecular profiling of low grade serous ovarian tumours identifies novel candidate driver genes. Oncotarget. 2015;6(35):37663-77.

20. Xing D, Suryo Rahmanto Y, Zeppernick F, Hannibal CG, Kjaer SK, Vang R, Shih IM, Wang TL. Mutation of NRAS is a rare genetic event in ovarian low-grade serous carcinoma. Hum Pathol. 2017;68:87-91.

21. Hsu CY, Bristow R, Cha MS, Wang BG, Ho CL, Kurman RJ, Wang TL, Shih le $M$. Characterization of active mitogen-activated protein kinase in ovarian serous carcinomas. Clin Cancer Res. 2004; 10:6432-6.

22. Farley J, Brady WE, Vathipadiekal V, Lankes HA, Coleman R, Morgan MA, Mannel R, Yamada SD, Mutch D, Rodgers WH, Birrer M, Gershenson DM. Selumetinib in women with recurrent low-grade serous carcinoma of the ovary or peritoneum: an open-label, single-arm, phase 2 study. Lancet Oncol. 2013;14:134-40.

23. Han C, Bellone S, Zammataro L, Schwartz PE, Santin AD. Binimetinib (MEK162) in recurrent low-grade serous ovarian cancer resistant to chemotherapy and hormonal treatment. Gynecol Oncol Rep. 2018;25:41-4.
24. Fernandez ML, DiMattia GE, Dawson A, Bamford S, Anderson S, Hennessy BT, Anglesio MS, Shepherd TG, Salamanca C, Hoenisch J, Tinker A, Huntsman DG, Carey MS. Differences in MEK inhibitor efficacy in molecularly characterized low-grade serous ovarian cancer cell lines. Am J Cancer Res. 2016:6:2235-51.

25. Chahal M, Pleasance E, Grewal J, Zhao E, Ng T, Chapman E, Jones MR, Shen Y, Mungall KL, Bonakdar M, Taylor GA, Ma Y, Mungall AJ, Moore RA, Lim H, Renouf D, Yip S, Jones SJM, Marra MA, Laskin J. Personalized oncogenomic analysis of metastatic adenoid cystic carcinoma: using whole-genome sequencing to inform clinical decision-making. Cold Spring Harb Mol Case Stud. 2018;4:a002626.

26. Tomkinson H, McBride E, Martin P, Lisbon E, Dymond AW, Cantarini M, So K, Holt D. Comparison of the pharmacokinetics of the phase II and phase III capsule formulations of selumetinib and the effects of food on exposure: results from two randomized crossover trials in healthy male subjects. Clin Ther. 2017;39(2260-2275):e2261.

27. Infante JR, Papadopoulos KP, Bendell JC, Patnaik A, Burris HA 3rd, Rasco D, Jones SF, Smith L, Cox DS, Durante M, Bellew KM, Park JJ, Le NT, Tolcher AW. A phase $1 \mathrm{~b}$ study of trametinib, an oral Mitogen-activated protein kinase kinase (MEK) inhibitor, in combination with gemcitabine in advanced solid tumours. Eur J Cancer. 2013;49:2077-85.

28. Tibes R, Qiu Y, Lu Y, Hennessy B, Andreeff M, Mills GB, Kornblau SM. Reverse phase protein array: validation of a novel proteomic technology and utility for analysis of primary leukemia specimens and hematopoietic stem cells. Mol Cancer Ther. 2006;5:2512-21.

29. Aslan O, Cremona M, Morgan C, Cheung LW, Mills GB, Hennessy BT. Preclinical evaluation and reverse phase protein array-based profiling of PI3K and MEK inhibitors in endometrial carcinoma in vitro. BMC Cancer. 2018;18:168.

30. Wiegand KC, Hennessy BT, Leung S, Wang Y, Ju Z, McGahren M, Kalloger SE, Finlayson S, Stemke-Hale K, Lu Y, Zhang F, Anglesio MS, Gilks B, Mills GB, Huntsman DG, Carey MS. A functional proteogenomic analysis of endometrioid and clear cell carcinomas using reverse phase protein array and mutation analysis: protein expression is histotype-specific and loss of ARID1A/BAF250a is associated with AKT phosphorylation. BMC Cancer. 2014:14:120.

31. Tusher VG, Tibshirani R, Chu G. Significance analysis of microarrays applied to the ionizing radiation response. Proc Natl Acad Sci USA. 2001;98:5116-21.

32. Chou TC, Talalay P. Quantitative analysis of dose-effect relationships: the combined effects of multiple drugs or enzyme inhibitors. Adv Enzyme Regul. 1984;22:27-55.

33. Corcoran RB, Andre T, Atreya CE, Schellens JHM, Yoshino T, Bendell JC, Hollebecque A, McRee AJ, Siena S, Middleton G, Muro K, Gordon MS, Tabernero J, Yaeger R, O'Dwyer PJ, Humblet Y, De Vos F, Jung AS, Brase JC, Jaeger S, Bettinger S, Mookerjee B, Rangwala F, Van Cutsem E. Combined BRAF, EGFR, and MEK inhibition in patients with BRAF(V600E)-mutant colorectal cancer. Cancer Discov. 2018;8:428-43.

34. Kim JY, Welsh EA, Fang B, Bai Y, Kinose F, Eschrich SA, Koomen JM, Haura EB. Phosphoproteomics reveals MAPK inhibitors enhance MET- and EGFR-driven AKT signaling in KRAS-mutant lung cancer. Mol Cancer Res. 2016;14:1019-29.

35. Li J, Sordella R, Powers S. Effectors and potential targets selectively upregulated in human KRAS-mutant lung adenocarcinomas. Sci Rep. 2016;6:27891

36. Kim CW, Asai D, Kang JH, Kishimura A, Mori T, Katayama Y. Reversal of efflux of an anticancer drug in human drug-resistant breast cancer cells by inhibition of protein kinase Calpha (PKCalpha) activity. Tumour Biol. 2016;37:1901-8.

37. Muscella A, Vetrugno C, Antonaci G, Cossa LG, Marsigliante S. PKC-delta/ PKC-alpha activity balance regulates the lethal effects of cisplatin. Biochem Pharmacol. 2015;98:29-40.

38. Momeny M, Yousefi H, Eyvani H, Moghaddaskho F, Salehi A, Esmaeil F, Alishahi Z, Barghi F, Vaezijoze S, Shamsaiegahkani S, Zarrinrad G, Sankanian G, Sabourinejad Z, Hamzehlou S, Bashash D, Aboutorabi ES, Ghaffari P, Dehpour AR, Tavangar SM, Tavakkoly-Bazzaz J, Alimoghaddam K, Ghavamzadeh A, Ghaffari SH. Blockade of nuclear factor-kappaB (NFkappaB) pathway inhibits growth and induces apoptosis in chemoresistant ovarian carcinoma cells. Int J Biochem Cell Biol. 2018;99:1-9.

39. Kankia IH, Khalil HS, Langdon SP, Moult PR, Bown JL, Deeni YY. NRF2 regulates HER1 signaling pathway to modulate the sensitivity of 
ovarian cancer cells to lapatinib and erlotinib. Oxid Med Cell Longev. 2017;2017:1864578.

40. Yeo WL, Riely GJ, Yeap BY, Lau MW, Warner JL, Bodio K, Huberman MS, Kris MG, Tenen DG, Pao W, Kobayashi S, Costa DB. Erlotinib at a dose of $25 \mathrm{mg}$ daily for non-small cell lung cancers with EGFR mutations. J Thorac Oncol. 2010;5:1048-53.

41. Barras D. BRAF mutation in colorectal cancer: an update. Biomark Cancer. 2015;7:9-12.

42. Safaee Ardekani G, Jafarnejad SM, Tan L, Saeedi A, Li G. The prognostic value of BRAF mutation in colorectal cancer and melanoma: a systematic review and meta-analysis. PLoS ONE. 2012;7:e47054.

43. Cheng Y, Tian H. Current development status of MEK inhibitors. Molecules. 2017;22(10):1551.

44. Grisham R, Moore KN, Gordon MS, Harb W, Cody GR, Halpenny DF, Makker V, Aghajanian C. Phase Ib study of binimetinib with paclitaxel in patients with platinum-resistant ovarian cancer: final results, potential biomarkers, and extreme responders. Clin Cancer Res. 2018. https://doi. org/10.1158/1078-0432.CCR-18-0494.

45. Gershenson DM, Sun CC, Bodurka D, Coleman RL, Lu KH, Sood AK, Deavers M, Malpica AL, Kavanagh JJ. Recurrent low-grade serous ovarian carcinoma is relatively chemoresistant. Gynecol Oncol. 2009;114:48-52.

46. Grisham RN, Sylvester BE, Won H, McDermott G, DeLair D, Ramirez R, Yao Z, Shen R, Dao F, Bogomolniy F, Makker V, Sala E, Soumerai TE, Hyman DM, Socci ND, Viale A, Gershenson DM, Farley J, Levine DA, Rosen N, Berger MF, Spriggs DR, Aghajanian CA, Solit DB, lyer G. Extreme outlier analysis identifies occult mitogen-activated protein kinase pathway mutations in patients with low-grade serous ovarian cancer. J Clin Oncol. 2015;33(34):4099-105.

47. Takekuma M, Wong KK, Coleman RL. A long-term surviving patient with recurrent low-grade serous ovarian carcinoma treated with the MEK1/2 inhibitor, selumetinib. Gynecol Oncol Res Pract. 2016:3:5.

48. Altman AD, Nelson GS, Ghatage P, McIntyre JB, Capper D, Chu P, Nation $J G$, Karnezis AN, Han G, Kalloger SE, Kobel M. The diagnostic utility of TP53 and CDKN2A to distinguish ovarian high-grade serous carcinoma from low-grade serous ovarian tumors. Mod Pathol. 2013;26:1255-63.

49. Kuo KT, Guan B, Feng Y, Mao TL, Chen X, Jinawath N, Wang Y, Kurman RJ, Shih le M, Wang TL. Analysis of DNA copy number alterations in ovarian serous tumors identifies new molecular genetic changes in low-grade and high-grade carcinomas. Cancer Res. 2009;69:4036-42.
50. McIntyre JB, Rambau PF, Chan A, Yap S, Morris D, Nelson GS, Kobel M. Molecular alterations in indolent, aggressive and recurrent ovarian lowgrade serous carcinoma. Histopathology. 2017;70:347-58.

51. Prahallad A, Sun C, Huang S, Di Nicolantonio F, Salazar R, Zecchin D, Beijersbergen RL, Bardelli A, Bernards R. Unresponsiveness of colon cancer to BRAF(V600E) inhibition through feedback activation of EGFR. Nature. 2012:483:100-3.

52. Whittaker SR, Cowley GS, Wagner S, Luo F, Root DE, Garraway LA. Combined Pan-RAF and mek inhibition overcomes multiple resistance mechanisms to selective RAF inhibitors. Mol Cancer Ther. 2015;14:2700-11.

53. Carter CA, Rajan A, Keen C, Szabo E, Khozin S, Thomas A, Brzezniak C, Guha U, Doyle LA, Steinberg SM, Xi L, Raffeld M, Tomita Y, Lee MJ, Lee S, Trepel JB, Reckamp KL, Koehler S, Gitlitz B, Salgia R, Gandara D, Vokes E, Giaccone G. Selumetinib with and without erlotinib in KRAS mutant and KRAS wild-type advanced nonsmall-cell lung cancer. Ann Oncol. 2016;27:693-9.

54. Corcoran RB, Ebi H, Turke AB, Coffee EM, Nishino M, Cogdill AP, Brown RD, Della Pelle P, Dias-Santagata D, Hung KE, Flaherty KT, Piris A, Wargo JA, Settleman J, Mino-Kenudson M, Engelman JA. EGFR-mediated reactivation of MAPK signaling contributes to insensitivity of BRAF mutant colorectal cancers to RAF inhibition with vemurafenib. Cancer Discov. 2012;2:227-35.

55. Chapman PB, Hauschild A, Robert C, Haanen JB, Ascierto P, Larkin J, Dummer R, Garbe C, Testori A, Maio M, Hogg D, Lorigan P, Lebbe C, Jouary T, Schadendorf D, Ribas A, O'Day SJ, Sosman JA, Kirkwood JM, Eggermont AM, Dreno B, Nolop K, Li J, Nelson B, Hou J, Lee RJ, Flaherty KT, McArthur GA, Group B-S. Improved survival with vemurafenib in melanoma with BRAF V600E mutation. N Engl J Med. 2011;364:2507-16.

56. Kopetz S, Desai J, Chan E, Hecht JR, O'Dwyer PJ, Maru D, Morris V, Janku F, Dasari A, Chung W, Issa JP, Gibbs P, James B, Powis G, Nolop KB, Bhattacharya S, Saltz L. Phase II pilot study of vemurafenib in patients with metastatic BRAF-mutated colorectal cancer. J Clin Oncol. 2015;33:4032-8.

57. Grisham RN, lyer G, Garg K, Delair D, Hyman DM, Zhou Q, lasonos A, Berger MF, Dao F, Spriggs DR, Levine DA, Aghajanian C, Solit DB. BRAF mutation is associated with early stage disease and improved outcome in patients with low-grade serous ovarian cancer. Cancer. 2013;119:548-54.

58. Wong KK, Tsang YT, Deavers MT, Mok SC, Zu Z, Sun C, Malpica A, Wolf JK, Lu KH, Gershenson DM. BRAF mutation is rare in advanced-stage lowgrade ovarian serous carcinomas. Am J Pathol. 2010;177:1611-7.
Ready to submit your research? Choose BMC and benefit from:

- fast, convenient online submission

- thorough peer review by experienced researchers in your field

- rapid publication on acceptance

- support for research data, including large and complex data types

- gold Open Access which fosters wider collaboration and increased citations

- maximum visibility for your research: over $100 \mathrm{M}$ website views per year

At BMC, research is always in progress.

Learn more biomedcentral.com/submissions 